\title{
Long-lasting Decrease in Viremia In Chronically SIVmac25 I-infected Macaques After Therapeutic Immunization With Combinations Of DNA Vectors
} George N Pavlakis*‡

\author{
Address: National Cancer Institute, Frederick, MD \\ Email: George N Pavlakis* - pavlakis@ncifcrf.gov \\ * Corresponding author ‡Presenting author
}

\author{
from 2005 International Meeting of The Institute of Human Virology \\ Baltimore, USA, 29 August - 2 September 2005 \\ Published: 8 December 2005 \\ Retrovirology 2005, 2(Suppl I):SI 40 doi:I0.I I86/I742-4690-2-SI-SI 40
}

We explored therapeutic immunization of ART-treated SIVmac251 infected rhesus macaques using a new generation of optimized DNA-based vaccine vectors that produce either secreted or intracellularly degraded SIV antigens. Macaques infected for 15-70 wks were treated with a combination of 3 drugs (ART) for 13-23 wks. During this time, the animals were immunized via the IV route with the SIV DNAs and then released from ART. Macaques receiving DNA showed a significant decrease in viral load for long periods after therapy termination compared to controls $(\mathrm{p}<0.001)$. DNA vaccination, but not ART alone, led to substantial decreases in viremia. Half of the animals (6/12) continue to control viremia levels for 2 years. Cellular immune responses were immediately boosted strongly by DNA vaccination and persisted despite lower virus loads. Thus, the combination of novel forms of DNA vaccines administered during ART treatment induced an immune response able to persistently decrease viremia after removal of ART. These DNA vectors used as therapeutic vaccine may be beneficial either alone or in combination with other vaccine modalities as an addition to antiretroviral treatment. 